\title{
Predictors of 90-day Colonic Diverticular Recurrent Bleeding and Readmission
}

\author{
Tomoyuki Kitagawa, Yasumi Katayama, Ikuhiro Kobori, Yo Fujimoto and Masaya Tamano
}

\begin{abstract}
:
Objective Colonic diverticular bleeding often recurs, and readmissions are common. The aim of this study was to identify predictors of colonic diverticular recurrent bleeding and readmission within 90 days.

Methods Subjects comprised 144 patients diagnosed with colonic diverticular bleeding who received inpatient hospital care between January 2012 and June 2017. A retrospective comparative study was carried out regarding the clinical characteristics during the hospital stay by dividing the cases into 2 groups: patients with recurrent bleeding requiring readmission within 90 days $(\mathrm{n}=17)$ and patients without recurrent bleeding $(\mathrm{n}=127)$.

Results A univariate analysis showed that recurrent bleeding and readmission were significantly more frequent among cases with hypovolemic shock on admission ( $\mathrm{p}=0.009$ ), blood transfusion during hospitalization $(\mathrm{p}=0.029)$, and hyperlipidemia $(\mathrm{p}=0.020)$ than among others. Shock on admission (odds ratio, 5.118; 95\% confidence interval, 1.168-22.426, $\mathrm{p}=0.030$ ) remained a significant predictor on a multivariate analysis.

Conclusion Shock may predict recurrent colonic diverticular bleeding and readmission within 90 days. Careful and adequate endoscopic hemostasis is recommended for patients showing shock on admission.
\end{abstract}

Key words: colon, diverticulum, gastrointestinal hemorrhage, patient readmission, shock

(Intern Med 58: 2277-2282, 2019)

(DOI: 10.2169/internalmedicine.2276-18)

\section{Introduction}

Diverticular disease is the most common cause of massive lower gastrointestinal bleeding (1). Although the prevalence of colonic diverticulosis in Japan is reportedly $13-28 \%$, the incidence of colonic diverticulosis is on the rise in Japan, due to the aging of society and the westernization of dietary habits. Colonic diverticular bleeding and colonic diverticulitis, as complications of this pathology, therefore also seem likely to increase. In fact, an increasing trend in diverticular bleeding has already been reported (2).

Approximately $40 \%$ of all cases of lower gastrointestinal bleeding are believed to be caused by hemorrhaging from colonic diverticula (3), often appearing with the sudden occurrence of fresh blood in the stool, necessitating immediate medical attention. Colonic diverticulosis is a state in which part of the intestinal tract wall of the colon protrudes and the colonic mucosa becomes sac-shaped. Colonic diverticu- lar bleeding is believed to be caused when the vascular intima of an artery directly inside the diverticular lumen thickens and becomes brittle due to repeated mechanical stimulation (4). However, no clear conclusion has been reached regarding the relationship between hypertension (5) and arteriosclerosis-related factors such as hyperlipidemia, diabetes, ischemic heart disease, and cerebrovascular disease (6), which are involved in the brittleness of arterial walls and colonic diverticular bleeding.

In recent years, nonsteroidal anti-inflammatory drugs (NSAIDs) have seen frequent use due to their strong antiinflammatory effects and analgesic actions. Mucosal lesions of the upper gastrointestinal tract are a well-known side effect of NSAIDs, but side effects of the lower digestive tract are garnering attention, with reports mentioning that complications such as diverticulitis (7) and diverticular bleeding $(7,8)$ tend to occur when NSAIDs are administered for colonic diverticulosis. Furthermore, some reports have mentioned obesity as a risk factor for diverticular disease of the

Department of Gastroenterology, Dokkyo Medical University Saitama Medical Center, Japan

Received: October 17, 2018; Accepted: January 16, 2019; Advance Publication by J-STAGE: May 22, 2019

Correspondence to Dr. Tomoyuki Kitagawa, t-kita@dokkyomed.ac.jp 
colon (9). Endoscopic treatments for colonic diverticular bleeding include injection of epinephrine $(10,11)$, heater probe coagulation (12), and endoclip placement (13). However, recurrent bleeding may require frequent endoscopic treatment. In fact, recurrent bleeding and readmission often occur in cases of colonic diverticular bleeding $(14,15)$. As recurrent bleeding occurs at various times, the risk factors in each period need to be considered. In recent years, the increased medical costs associated with readmission have come to be viewed as a major issue. Jencks et al. (16) analyzed Medicare claims data and reported that $19.6 \%$ of patients released from hospital were readmitted within 30 days, with $34.0 \%$ readmitted within 90 days.

Investigating the risk factors for recurrent bleeding is therefore expected to prove helpful in clinical practice and important in terms of medical costs.

\section{Materials and Methods}

\section{Patients and data collection}

Subjects were 144 patients diagnosed with colonic diverticular hemorrhaging who received inpatient hospital care at Dokkyo Medical University Saitama Medical Center between January 2012 and June 2017. The endoscopic database and medical records of patients in our hospital were reviewed, and data were extracted. To clarify the risk factors for recurrent bleeding and readmission, a retrospective comparative study was carried out regarding the clinical characteristics during the hospital stay by dividing cases into 2 groups: a group showing recurrent bleeding and readmission within 90 days after discharge and a group without recurrent bleeding. Examination items were age, sex, body mass index (BMI), history of previous diverticular bleeding, concentration of hemoglobin $(\mathrm{Hb})$ on admission, presence of blood transfusion, presence of hypovolemic shock on admission, length of hospital stay, underlying diseases (hypertension, diabetes, hyperlipidemia, chronic renal failure, ischemic heart disease, cerebrovascular disease), oral medicines (nonsteroidal anti-inflammatory drugs (NSAIDs), antiplatelets, aspirin, anticoagulants, steroids), presence of active bleeding on colonoscopy, site of bleeding, and endoscopic treatment.

\section{Definition}

The diagnosis of colonic diverticular bleeding was made based on the criteria reported by Jensen et al. (10). A patient with hematochezia was diagnosed with colonic diverticular bleeding if active bleeding from a diverticulum was observed and/or blood clots were found in the colon with diverticula with no blood in the terminal ileum and no other demonstrable cause of bleeding. The need for blood transfusion was determined by the primary physician based on the condition and $\mathrm{Hb}$ level of each patient. Hypovolemic shock was defined as systolic blood pressure $\leq 90 \mathrm{mmHg}$ and/or shock index $(\mathrm{SI}) \geq 1$ (17) and the exclusion of cardiogenic shock, distributive shock and obstructive shock. The BMI was calculated as the weight in kilograms divided by the square of the height in meters.

\section{Colonoscopy}

All patients underwent colonoscopy after admission. Urgent cases such as those with active bleeding underwent colonoscopy either without bowel preparation or with enema only. Patients able to tolerate elective procedures underwent colonoscopy with bowel preparation using lavage solution containing polyethylene-glycol. If colonoscopy revealed active bleeding from a diverticulum, endoscopic hemostasis was performed using a hemoclip (18). Clips were placed across the margin of the diverticulum or in sequential zipper fashion. Cases with stigmata of recent hemorrhaging were treated with fasting and bed rest. Patients were started on a liquid diet once bleeding symptoms were no longer apparent and were discharged if no bleeding was identified when switched to solid diet.

\section{Definition of recurrent bleeding}

Recurrent bleeding was confirmed directly by colonoscopy, and only bleeding from the same diverticulum that had previously shown bleeding was defined as recurrent bleeding. Cases in which the bleeding point could not be confirmed by colonoscopy were not regarded as recurrent bleeding in this study, and were included in the without recurrent bleeding group.

\section{Statistical analyses}

Data were evaluated using descriptive statistical methods (mean \pm standard deviation). Numerical data were compared using the Mann-Whitney U-test, and categorical data were compared using the chi-squared test or Fisher's exact probability test, as appropriate. Comparisons of the recurrencefree period were performed using a log-rank test with the Kaplan-Meier method. Values of $\mathrm{p}<0.05$ were considered statistically significant. All statistical analyses were performed using SPSS version 24 software (SPSS, Chicago, USA).

\section{Results}

\section{Patient characteristics}

Table 1 shows the clinical features and characteristics of the 144 patients. The 144 patients diagnosed with colonic diverticular hemorrhage comprised 87 men (60.4\%) and 57 women $(39.6 \%)$, with a mean age of $73.8 \pm 10.5$ years. The mean BMI was $22.7 \pm 3.8 \mathrm{~kg} / \mathrm{m}^{2}$. Blood transfusion was required by 34 patients $(23.6 \%)$, and 13 patients $(9.0 \%)$ were found to have experienced shock. Blood transfusion was performed in $8(61.5 \%)$ of those 13 shock patients. Active bleeding was identified in 14 patients $(9.7 \%)$. Seventeen patients $(11.8 \%)$ had recurrent bleeding and were readmitted within 90 days. Recurrent bleeding occurred after an average of $45.6 \pm 28.7$ days. None of the patients required tran- 
Table 1. Clinical Features and Characteristics of the 144 Patients and Comparison of Clinical Features between Patients with and without Recurrent Bleeding.

\begin{tabular}{|c|c|c|c|c|}
\hline & $\begin{array}{l}\text { All cases } \\
(n=144)\end{array}$ & $\begin{array}{l}\text { With recurrent bleeding } \\
\qquad(\mathrm{n}=17)\end{array}$ & $\begin{array}{l}\text { Without recurrent bleeding } \\
\qquad(\mathrm{n}=127)\end{array}$ & $\mathrm{p}$ value \\
\hline Male:Female & $87(60.4): 57(39.6)$ & $11(64.7): 6(35.3)$ & $76(59.8): 51(40.2)$ & 0.700 \\
\hline Age, years & $73.8 \pm 10.5$ & $75.9 \pm 8.5$ & $73.6 \pm 10.8$ & 0.401 \\
\hline BMI, kg/m² & $22.7 \pm 3.8$ & $23.7 \pm 5.5$ & $22.6 \pm 3.6$ & 0.804 \\
\hline $\mathrm{Hb}$ on admission, $\mathrm{g} / \mathrm{dL}$ & $10.3 \pm 2.3$ & $9.5 \pm 2.1$ & $10.4 \pm 2.3$ & 0.105 \\
\hline History of previous diverticular bleeding & $43(29.9)$ & $7(41.2)$ & $36(28.3)$ & 0.278 \\
\hline Blood transfusion & $34(23.6)$ & $8(47.1)$ & $26(20.5)$ & 0.029 \\
\hline Shock on admission & $13(9.0)$ & $5(29.4)$ & $8(6.3)$ & 0.009 \\
\hline Length of hospital stay, days & $12.2 \pm 8.4$ & $12.7 \pm 4.7$ & $12.1 \pm 8.8$ & 0.239 \\
\hline \multicolumn{5}{|l|}{ Comorbidities } \\
\hline Hypertension & $63(43.8)$ & $6(35.2)$ & $57(44.9)$ & 0.454 \\
\hline Diabetes mellitus & $32(22.2)$ & $2(11.8)$ & $30(23.6)$ & 0.362 \\
\hline Hyperlipidemia & $27(18.8)$ & $7(41.2)$ & $20(15.7)$ & 0.020 \\
\hline Chronic renal failure & $12(8.3)$ & $2(11.8)$ & $10(7.9)$ & 0.635 \\
\hline Ischemic heart disease & $20(18.9)$ & $6(35.3)$ & $31(24.4)$ & 0.378 \\
\hline Cerebrovascular disease & $16(11.1)$ & $0(0.0)$ & $16(12.6)$ & 0.217 \\
\hline \multicolumn{5}{|l|}{ Oral medication } \\
\hline NSAIDs & $7(4.9)$ & $1(5.9)$ & $6(4.7)$ & 0.593 \\
\hline Antiplatelets & $61(42.4)$ & $8(47.1)$ & $53(41.7)$ & 0.676 \\
\hline Aspirin & $44(31.4)$ & $7(41.2)$ & $37(29.1)$ & 0.311 \\
\hline Anticoagulants & $25(17.3)$ & $1(5.9)$ & $24(18.9)$ & 0.307 \\
\hline Steroids & $4(2.8)$ & $1(5.9)$ & $3(2.4)$ & 0.398 \\
\hline \multicolumn{5}{|l|}{ SRH diverticula } \\
\hline Active bleeding & $14(9.7)$ & $4(23.5)$ & $10(7.9)$ & 0.064 \\
\hline Non-bleeding visible vessel & $1(0.69)$ & $0(0.0)$ & $1(0.79)$ & 1.000 \\
\hline Adherent clot & $10(6.9)$ & $0(0.0)$ & $10(7.9)$ & 0.607 \\
\hline
\end{tabular}

Data are presented as mean \pm standard deviation or number $(\%)$.

BMI: body mass index, Hb: hemoglobin, NSAID: nonsteroidal anti-inflammatory drug, SRH: stigmata recent hemorrhage

Table 2. Predictors of Recurrent Bleeding from Logistic Regression in Multivariate Analysis.

\begin{tabular}{lccc}
\hline & Odds ratio & 95\%CI & p value \\
\hline Shock on admission & 5.118 & $1.168-22.429$ & 0.030 \\
Blood transfusion & 1.678 & $0.478-5.860$ & 0.420 \\
Hyperlipidemia & 3.022 & $0.937-9.746$ & 0.064 \\
Active bleeding on first colonoscopy & 3.157 & $0.700-14.247$ & 0.135 \\
\hline CI: confidence interval & & &
\end{tabular}

scatheter arterial embolization or surgery.

\section{A comparative analysis of the risk factors of diver- ticular recurrent bleeding}

To identify risk factors for diverticular recurrent bleeding, we compared the group with recurrent bleeding within 90 days $(n=17)$ and the group without recurrent bleeding $(n=$ 127). The results are shown in Table 1. No significant differences were identified between groups in the sex, mean age, mean BMI, or hemoglobin levels on admission, but recurrent bleeding was significantly more frequent among patients who required a transfusion than among those not requiring a transfusion $(47.1 \%$ vs. $20.5 \%, \mathrm{p}=0.029)$ and patients presenting with hypovolemic shock on admission than among those without shock $(29.4 \%$ vs. $6.3 \%, \mathrm{p}=0.009)$. On comparing concurrent underlying diseases and oral medicines, recurrent bleeding was significantly more frequent among patients with hyperlipidemia than among those without it $(41.2 \%$ vs. $15.7 \%, \mathrm{p}=0.020)$. On comparing endoscopy-related factors, the number of patients in whom active bleeding was seen during colonoscopy did not differ significantly between the groups. However, a trend toward recurrent bleeding was detected. A multivariate analysis identified shock (odds ratio, 5.118; 95\% confidence interval, $1.168-22.426, \mathrm{p}=0.030$ ) as an independent risk factor for recurrent bleeding (Table 2).

We compared patients with and without hemostasis in order to examine the effect of hemostasis (Table 3) and showed that patients who underwent hemostasis were significantly more frequent among those who required transfu- 
Table 3. Comparison of Clinical Features between Patients with and without Hemostasis.

\begin{tabular}{lccc}
\hline & $\begin{array}{c}\text { With hemostasis } \\
(\mathrm{n}=14)\end{array}$ & $\begin{array}{c}\text { Without hemostasis } \\
(\mathrm{n}=130)\end{array}$ & $\mathrm{p}$ value \\
\hline Male:Female & $11(78.6): 3(21.4)$ & $76(58.5): 54(41.5)$ & 0.144 \\
Age, years & $70.0 \pm 11.2$ & $74.3 \pm 10.4$ & 0.108 \\
BMI, kg/m & $22.5 \pm 3.6$ & $22.7 \pm 3.9$ & 0.917 \\
Hb on admission, g/dL & $10.0 \pm 2.5$ & $10.4 \pm 2.3$ & 0.473 \\
Blood transfusion & $8(57.1)$ & $26(20.0)$ & 0.005 \\
Shock on admission & $1(7.1)$ & $12(9.2)$ & 1.000 \\
Length of hospital stay, days & $12.9 \pm 5.5$ & $12.1 \pm 8.7$ & 0.104 \\
Recurrent bleeding and & $4(28.6)$ & $13(10.0)$ & 0.064 \\
readmission within 90 days & & & \\
\hline
\end{tabular}

Data are presented as mean \pm standard deviation or number $(\%)$

BMI: body mass index, Hb: hemoglobin

Table 4. Comparison of Clinical Features between Patients with and without Recurrent Bleeding among Conservative Therapy Group $(n=130)$.

\begin{tabular}{lccc}
\hline & $\begin{array}{c}\text { With recurrent bleeding } \\
(\mathrm{n}=13)\end{array}$ & $\begin{array}{c}\text { Without recurrent bleeding } \\
(\mathrm{n}=117)\end{array}$ & $\mathrm{p}$ value \\
\hline Male:Female & $8(61.5): 5(38.5)$ & $68(58.1): 49(41.9)$ & 0.812 \\
Age, years & $78.5 \pm 4.8$ & $73.8 \pm 10.8$ & 0.167 \\
BMI, $\mathrm{kg} / \mathrm{m}^{2}$ & $24.1 \pm 5.4$ & $22.6 \pm 3.7$ & 0.701 \\
Hb on admission, g/dL & $9.5 \pm 2.4$ & $10.5 \pm 2.3$ & 0.206 \\
History of previous diverticular bleeding & $6(46.2)$ & $31(26.5)$ & 0.192 \\
Blood transfusion & $5(38.5)$ & $21(17.9)$ & 0.135 \\
Shock on admission & $4(30.8)$ & $8(6.8)$ & 0.019 \\
Length of hospital stay, days & $11.8 \pm 4.0$ & $12.1 \pm 9.1$ & 0.505 \\
\hline
\end{tabular}

Data are presented as mean \pm standard deviation or number $(\%)$.

BMI: body mass index, Hb: hemoglobin

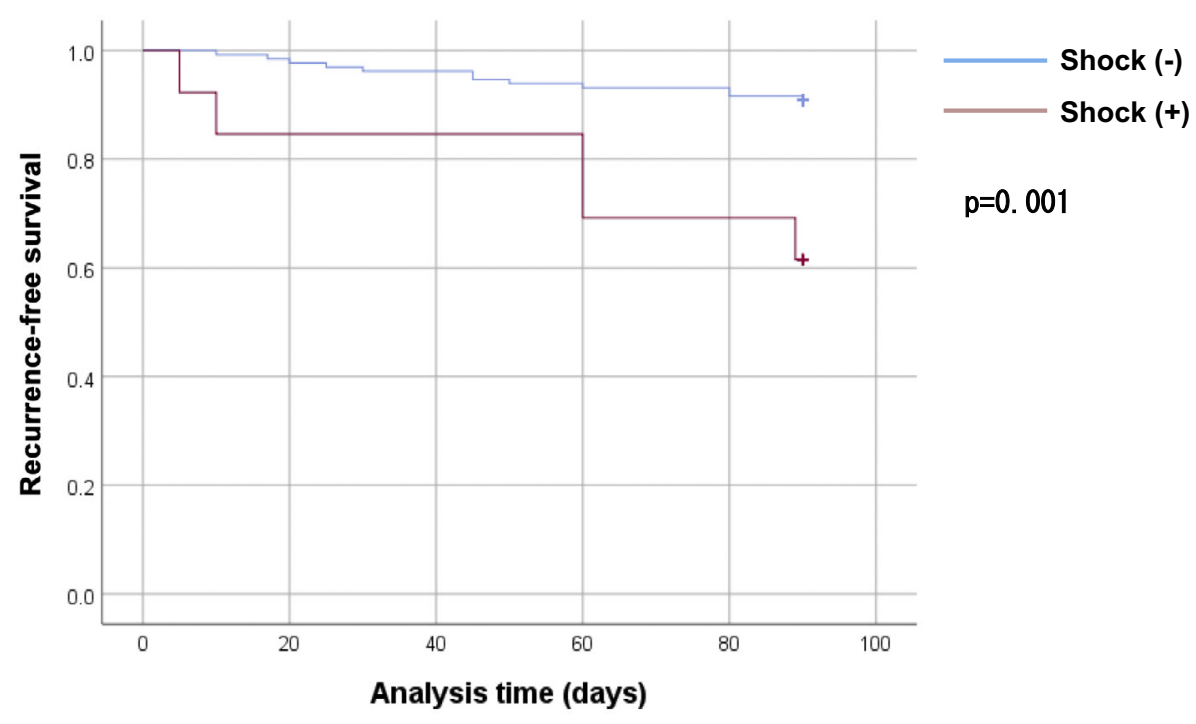

Figure. The recurrence-free period in patients with and without shock.

sion than among those not requiring transfusion $(57.1 \%$ vs. $20.0 \%, \mathrm{p}=0.005)$. Regarding recurrent bleeding and readmission, no significant differences were apparent between the groups with and without hemostasis $(28.6 \%$ vs. $10.0 \%, \mathrm{p}=$ 0.064). We conducted a similar comparative study for 130 cases with conservative treatment (Table 4). Recurrent bleeding was significantly more frequent among patients presenting with hypovolemic shock on admission than among those without shock $(30.8 \%$ vs. $6.8 \%, \mathrm{p}=0.019)$. Figure shows the Kaplan-Meier curves comparing recurrent bleeding and read- 
mission rates between the shock and no-shock groups. The shock group showed higher frequencies of recurrent bleeding and readmission than the no-shock group (log-rank $\mathrm{p}=$ $0.001)$.

\section{Discussion}

The present study showed that shock on admission, blood transfusion, hyperlipidemia, and active bleeding on colonoscopy were risk factors for recurrent bleeding and readmission within 90 days. However, in a multivariate analysis, only shock remained an independent risk factor.

Joaquim et al. (19) reported that an older age and chronic renal failure were risk factors, while Tanaka et al. (20) reported that ecurrent bleeding was more likely with a higher BMI than with a lower value. In the present study, the age, BMI, and chronic renal failure were not found to be risk factors associated with recurrent bleeding, possibly because the sample size of this study was too small to reveal these relationships. Increases in the number of patients and duration of follow-up would help clarify such issues and provide more reliable results. Our univariate analysis revealed hyperlipidemia to be a risk factor. As reported by Okamoto et al. (6), this suggests that arteriosclerosis may be associated with recurrent diverticular bleeding. Associations between oral medicines such as NSAIDs, aspirin, and antiplatelets with diverticular bleeding have also occasionally been reported (21-24), but the use of NSAIDs, aspirin, and antiplatelets was not a risk factor in the present study. One possible reason for this may be that the use of these agents was assessed at the time of admission, and the use of such drugs would generally have been suspended after control over bleeding was achieved and then restarted after hemostasis was confirmed. Furthermore, the usage rate of around 5\% for NSAIDs in this study cohort seems lower than in previous studies of colonic diverticular bleeding (23). NSAIDs have recently been suggested to cause gastrointestinal damage (25), which has prompted doctors in other departments to refrain from prescribing these agents.

Several investigations of risk factors for recurrent diverticular bleeding have been reported. This is the first study to report a relationship between SI and recurrent diverticular bleeding. Fujino et al. (26) reported that signs of shock are a risk factor for early recurrent bleeding. Pallor and the like are used as signs of shock, but the specific criteria may vary depending on the individual evaluator. In the present study, shock was clearly defined as a systolic blood pressure $\leq 90$ $\mathrm{mmHg}$ or $\mathrm{SI} \geq 1$, and the clinical significance was deemed to be high, since more specific criteria were used. These results indicate the possibility of massive hemorrhaging and recurrent bleeding in shock patients, patients who require blood transfusion, and patients who show active bleeding on endoscopy. Thicker blood vessels in the diverticula were thought to have ruptured in patients with substantial bleeding, and these patients were considered more susceptible to recurrent bleeding than no-shock patients, patients who do not require blood transfusion, and patients who do not show active bleeding on endoscopy.

According to the Kaplan-Meier curves, patients with shock experienced recurrent bleeding relatively early. In our study, the mean hospital stay was $12.2 \pm 8.4$ days, and patients with shock had better may have been admitted for a longer time to avoid recurrent bleeding after discharge. However, if reliable hemostasis with a low recurrent bleeding rate can be achieved, then extended hospitalization would be unnecessary.

Regarding hemostatic procedures, bleeding may not be adequately stopped with a conventional clip, so performing procedures with a high hemostatic effect, such as endoscopic band ligation (EBL), is preferable. According to the present results, shock patients showed relatively high frequencies of recurrent bleeding, regardless of hemostasis. In our study, a clip procedure was selected for hemostasis in all cases, but recurrent bleeding was seen in $28.6 \%$ of cases. The rate of recurrent bleeding is considered to be lower with EBL than with a clip. Setoyama et al. (27) reported a recurrent bleeding rate of $33.3 \%$ with the clip method, compared to $5.6 \%$ with EBL. Endoscopic hemostasis can be achieved using endoclips via the direct placement of endoclips onto the vessel or the closure of the diverticulum in a zipper fashion. In our study, all patients who underwent hemostasis were treated with clipping applied in a zipper-like fashion. Hemostasis procedures may thus have only been mildly effective.

Increased readmissions for repeated recurrent bleeding also lead to higher medical costs. Ito et al. (28) confirmed that medical costs increase with recurrent bleeding from colonic diverticular bleeding and blood transfusion. Preventing recurrent bleeding is thus deemed to be extremely important in terms of reducing medical costs. Ensuring successful hemostasis is critical for preventing recurrent bleeding and thereby reducing associated medical costs. Patients with shock should therefore be examined using modalities such as computed tomography in an effort to identify the location of bleeding.

Limitations of this study included the single-center, retrospective study design and the small sample size.

\section{Conclusion}

We found that hypovolemic shock may serve as a predictor of colonic diverticular recurrent bleeding within 90 days. Assessing the patient's condition using the SI on admission is important. Strict observation of the course, identification of the source of bleeding, and reliable hemostasis are advisable in shock patients and will lead to a reduction in medical costs. Further research will probably be necessary to confirm our results.

The authors state that they have no Conflict of Interest (COI). 


\section{References}

1. Adams JB, Margolin DA. Management of diverticular hemorrhage. Clin Colon Rectal Surg 22: 181-185, 2009.

2. Kinjo K, Matsui T, Hisabe T, et al. Increase in colonic diverticular hemorrhage and confounding factors. World J Gastrointest Pharmacol Ther 7: 440-446, 2016.

3. Zuckerman GR, Prakash C. Acute lower intestinal bleeding, 2: etiology, therapy, and outcomes. Gastrointest Endosc 49: 228-238, 1999.

4. Meyers MA, Alonso DR, Gray GF, Baer JW. Pathogenesis of bleeding colonic diverticula. Gastroenterology 71: 577-583, 1976.

5. Niikura R, Nagata N, Akiyama J, Shimbo T, Uemura N. Hypertension and concomitant arteriosclerotic disease are risk factor for colonic diverticular bleeding: a case-control study. Int J Colorectal Dis 27: 1137-1143, 2012.

6. Okamoto T, Watanabe H, Yamada A, Hirata Y, Yoshida H, Koike $\mathrm{K}$. The association between arteriosclerosis related diseases and diverticular bleeding. Int J Colorectal Dis 27: 1161-1166, 2012.

7. Strate LL, Liu YL, Huang ES, Giovannucci EL, Chan AT. Use of aspirin or nonsteroidal anti-inflammatory drugs increase risk for diverticulitis and diverticular bleeding. Gastroenterology 140: 1427-1433, 2011.

8. Foutch PG. Diverticular bleeding: are nonsteroidal antiinflammatory drugs risk factor for hemorrhage and can colonoscopy predict outcome for patient? Am J Gastoenterol 90: 17791784, 1995

9. Strate LL, Liu YL, Aldoori WH, et al. Obesity increase the risks of diverticulitis and diverticular bleeding. Gastroenterology 136: 115-122, 2009.

10. Jensen DM, Machicado GA, Jutabha R, Kovacs TO. Urgent colonoscopy for the diagnosis and treatment of severe diverticular hemorrhage. N Engl J Med 342: 78-82, 2000.

11. Ramirez FC, Johnson DA, Zierer ST, Walker GJ, Sanowski RA. Successful endoscopic hemostasis of bleeding colonic diverticular with epinephrine injection. Gastrointest Endosc 43: 167-170, 1996.

12. Savides TJ, Jensen DM. Colonoscopic hemostasis for recurrent diverticular hemorrhage associated with a visible vessel: a report of three cases. Gastrointest Endosc 40: 70-73, 1994.

13. Simpson PW, Nguyen MH, Lim JK, Soetiko RM. Use of endoclips in the treatment of massive colonic diverticular bleeding. Gastrointest Endosc 59: 433-437, 2004.

14. Jensen DM, Singh B, Jensen ME, Sun N, Carrico M. How often do patients rebleed during long-term follow-up after an initial documented diverticular hemorrhage? Gastroenterology 134: A 112-A113, 2001.

15. Koperna T, Kisser M, Reiner G, Schulz F. Diagnosis and treatment of bleeding colonic diverticula. Hepatogastroenterology 48: 702705, 2001.
16. Jencks SF, Williams MV, Coleman EA. Rehospitalizations among patients in the Medicare fee-for-service program. N Engl J Med 360: 1418-1428, 2009.

17. Mutschler M, Nienaber U, Münzberg M, et al. The Shock Index revisited - a fast guide to transfusion requirement? A retrospective analysis on 21,853 patients derived from the TraumaRegister DGU ${ }^{\circledR}$. Crit Care 17: R172, 2013.

18. Hokama A, Uehara T, Nakayoshi $T$, et al. Utility of endoscopic hemoclipping for colonic diverticular bleeding. Am J Gastroenterol 92: 543-546, 1997.

19. Joaquim N, Caldeira P, Antunes AG, Eusébio M, Guerreiro H. Risk factors for severity and recurrence of colonic diverticular bleeding. Rev Esp Enferm Dig 109: 3-9, 2017.

20. Tanaka Y, Motomura $Y$, Akahoshi $\mathrm{K}$, et al. Predictive factors for colonic diverticular rebleeding: a retrospective analysis of the clinical and colonoscopic features of 111 patients. Gut Liver 6: 334-338, 2012.

21. Taki M, Oshima T, Tozawa K, et al. Analysis of risk factors for colonic diverticular bleeding and recurrence. Medicine 96: e8090, 2017.

22. Niikura R, Nagata N, Yamada A, Akiyama J, Shimbo T, Uemura $\mathrm{N}$. Recurrence of colonic diverticular bleedingand associated risk factors. Colorectal Dis 14: 302-305, 2012.

23. Nagata N, Niikura R, Aoki $\mathrm{T}$, et al. Impact of discontinuing nonsteroidal antiinflammatory drugs on long-term recurrence in colonic diverticular bleeding. World J Gastroenterol 21: 1292-1298, 2015.

24. Nagata N, Niikura R, Aoki T, et al. Colonic diverticular hemorrhage associated with the use of nonsteroidal anti-inflammatory drugs, low-dose aspirin, antiplatelet drugs, and dual therapy. J Gastroenterol Hepatol 29: 1786-1793, 2014.

25. Bjarnason I, Scarpignato C, Holmgren E, Olszewski M, Rainsford $\mathrm{KD}$, Lanas A. Mechanisms of damage to the gastrointestinal tract From nonsteroidal anti-Inflammatory drugs. Gastroenterology 154: 500-514, 2018.

26. Fujino Y, Inoue Y, Onodera M, et al. Risk factors for early rebleeding and associated hospitalization in patients with colonic diverticular bleeding. Colorectal Dis 43: 982-986, 2013.

27. Setoyama T, Ishii N, Fujita Y. Endoscopic band ligation (EBL) is superior to endoscopic clipping for the treatment of colonic diverticular hemorrhage. Surg Endosc 25: 3574-3578, 2011.

28. Ito Y, Sakata Y, Yoshida H, et al. High cost of hospitalization for colonic diverticular bleeding depended on repeated bleeding and blood transfusion: analysis with diagnosis procedure combination data in Japan. Digestion 96: 76-80, 2017.

The Internal Medicine is an Open Access journal distributed under the Creative Commons Attribution-NonCommercial-NoDerivatives 4.0 International License. To view the details of this license, please visit (https://creativecommons.org/licenses/ by-nc-nd/4.0/).

(C) 2019 The Japanese Society of Internal Medicine

Intern Med 58: 2277-2282, 2019 